\title{
Evaluation of HER-2/neu Overexpression in Gastric Carcinoma using a Tissue Microarray
}

\author{
Nasser Rakhshani ${ }^{1,3}$, Elham Kalantari², Hadi Bakhti' ${ }^{1}$, Masoud Reza Sohrabi \\ Mitra Mehrazma ${ }^{2 *}$
}

\begin{abstract}
Background: Amplification and overexpression of human epidermal growth factor receptor 2 (HER2 / neu) oncogene has considerable prognostic value in breast and gastric cancers. This study aimed to evaluate the frequency, overexpression pattern, clinical significance, and concordance between the results for protein expression and gene amplification of HER-2/neu in gastric and gastro-esophageal junction carcinomas. Materials and Methods: In this study, 101 gastric tissue samples which were included in tissue microarray were immunohistochemically examined for overexpression of HER2/neu. Chromogenic in situ hybridization (CISH) was used for HER-2/neu amplification. The correlation of HER2/neu amplification with clinicopathological parameters was also assessed. In addition, concordance between CISH and IHC was detected. Results: This study demonstrated a significant difference in the overexpression of HER2/neu in gastric tumors. The overexpression of HER2/neu was significantly higher in intestinal type, poorly differentiated grade, large size $(5 \mathrm{~cm} \leq)$ and positive nodal involvement tumors (p-value $=0.041,0.015,0.038$ and 0.071 , respectively). Also, amplification of HER2/neu according to CISH test, had a significant positive correlation with tumor size and tumor type (p-value=0.018 and 0.058, respectively).Concordance between CISH and IHC was 76.9\% in 101 evaluable samples. Conclusions: IHC/CISH differences were attributed to basolateral membranous immunoreactivity of glandular cells resulting in incomplete membranous reactivity and/or a higher rate of tumor heterogeneity in gastric cancers compared to breast cancers. Therefore, this can be a potential marker for targeted therapy of malignant gastric tumors.
\end{abstract}

Keywords: Chromogenic in situ hybridization - gastric cancer - HER2 - immunohistochemistry - tissue microarray

Asian Pac J Cancer Prev, 15 (18), 7597-7602

\section{Introduction}

Human epidermal growth factor receptor 2 (HER2 /neu) oncogene has proved prognostic significance in breast cancer (Koeppen et al., 2001; Takehana et al., 2002). Amplification of HER-2/neu oncogene has become an important biomarker for identifying patients who respond to HER-2 targeted therapy using the humanized monoclonal antibody trastuzumab (Herceptin). The role of HER-2/neu in other histological types of human cancer is less well established. Ovarian, endometrial, salivary gland, lung, esophageal and gastric carcinomas have been reportedly HER-2/neu positive in different studies (Hetzel et al., 1992; Hirashima et al., 2001; Kiessling et al., 2002; Eltze et al., 2005; Schuell et al., 2006; Reichelt et al., 2007; Tapia et al., 2007; Thompson et al., 2011). There are a number of studies focusing on the overexpression of HER2 in gastric cancer. One study has reported that $24 \%$ of patients with gastro-oesophageal adenocarcinomas showed an overexpression of HER2 (Tanner et al.,
2005). In addition, other studies have shown HER2 as an important prognostic factor in gastric cancer (Allgayer et al., 2000; Ross and McKenna, 2001; Shinohara et al., 2002); and the positive rate of HER2 in gastric cancer is estimated to be between $15 \%$ and $20 \%$ (Tanner et al., 2005; Yano et al., 2006). However, there are some conflicting attitudes in this respect, and the association between HER 2 overexpression and poor prognosis is not confirmed in all studies (Ross and McKenna, 2001). HER2/neu positive tumors from these cancer types might be considered potential targets for anti-HER-2 therapy with trastuzumab (Herceptin ${ }^{\mathrm{TM}}$ ) (Slamon et al., 2001; Smith et al., 2007). Immunohistochemistry (IHC) to detect HER2 protein and chromogenic in situ hybridization (CISH) to detect gene amplification are standard validated testing methods used for trastuzumab treatment globally (Hofmann et al., 2008).

In this study, we examined the frequency of HER-2/neu overexpression in a series of 101 gastric and gastro esophageal junction carcinomas, using 
immunohistochemistry( IHC) staining and Chromogenic In Situ hybridization (CISH) for HER-2/neu on six blocks of tissue microarray (TMA) made from the donor formalin-fixed paraffin-embedded blocks containing tumors. We also evaluated any significant correlation of HER-2/neu positive gastric carcinomas with age, sex, location of the tumor (gastric vs. gastroesophageal junction), the histologic subtype of the tumor according to Lauren's classification (intestinal, diffuse or mixed), the pathologic grade (well, moderately or poorly differentiated), the pathologic stage, size of the tumor and the lymph node involvement. Among the HER2 IHC scoring systems for gastric cancer, the HercepTest ${ }^{\mathrm{TM}}$ (Dako Denmark A/S, Glostrup, Denmark) is frequently used to evaluate the patterns of membranous immunoreactivity on the tumor cells for HER2 overexpression. This scoring system is based on the intensity of reactivity, whether complete or incomplete, and the percentage of reactive cells. The patterns are scored as IHC 0 (negative), IHC $1+$ (negative), IHC 2+ (equivocal) or IHC $3+$ (positive) for HER2 overexpression. The samples scored as IHC $2+$ should be retested with FISH or CISH, according to the testing algorithm (Hanna and Kwok, 2006; Hofmann et al., 2008).

\section{Materials and Methods}

\section{Samples and tissue collection}

This was a retrospective study on 101 surgically treated gastric gastroesophageal carcinomas conducted in the pathology departments of two referral hospitals (Mehr and Milad) in Tehran, Iran. We selected 101 cases of gastric carcinoma with the presence of sufficient tumor in a single tissue block for the study.

All the cases had proved gastric carcinoma and underwent total or partial gastrectomy. Their pathology reports were available at the pathology departments of Mehr and Milad hospitals. We selected the paraffinembedded tissue blocks which contained enough tumor tissue. The clinicopathological data (age, sex, histological subtype, tumor location, tumor size, lymph node involvement and pathologic grade and stage) were obtained from the pathology reports of the pathology departments.

A new H\&E slide of each tumor was made and reviewed to determine the histological subtype according to Lauren`s classification (Choi et al., 2009).

\section{TMA construction}

A map of receiver block was prepared with coordinates for each sample to correctly identify the tumor. Under a light microscope, the areas of interest that were nonnecrotic and rich in tumor were marked on the whole hematoxylin and eosin stained (H\&E) section of each donor's block. TMAs were constructed using a manual tissue array instrument. Tissue microarray blocks (TMAs) were obtained with $1.5 \mathrm{~mm}$ diameter of representative areas of each case ( 3 cores of different areas of the tumor from a single tissue block). The cores were carefully selected on H\&E stained sections and inserted into new paraffin blocks using a TMA workstation (Minicore;
ALPHELYS, Plaisir, France). At last, the sections from these blocks were cut into $4 \mu \mathrm{m}$ thick sections. The sections were mounted on TMA Slides (Superfrost plus, Thermo Scientific, Germany). These TMA recipient blocks were once more sectioned and stained by the H\&E method to confirm the presence of enough tumor cells at each single core (Keymoosi et al., 2013; Mehrazma et al., 2013; Gheytanchi et al., 2014).

\section{Immunohistochemistry staining}

Overexpression of HER2 was analyzed using rabbit polyclonal ALDH1 antibody (Dako, Denmark) By immunohistochemistry method as described previously (Madjd et al., 2012). The positively charged slides created from the recipient TMA blocks were deparafinized, rehydrated and incubated for 15 minutes with $3 \%$ hydrogen peroxidase to inhibit endogenous peroxidase activity and were then heated in $0.01 \mathrm{~mol} / \mathrm{L}$ citrate buffer $(\mathrm{pH}=6.0)$ in a microwave oven for 5 minutes at $100^{\circ} \mathrm{C}$ for antigen retrieval.

After cooling down, the sections were incubated for 15 minutes in a blocking solution containing $10 \%$ normal goat serum in PBS. Her-2/neu was stained using HercepTest ${ }^{\mathrm{TM}}$ Kit (K5204) according to the manufacturer's instructions (DAKO). Detection was done by the Biotin-Streptavidin Amplified (B-SA) system with diaminobenzidine chromogen (DAB) as a routine protocol. Meyer's hematoxylin was used for counter staining. The slides were then dehydrated, cleared and mounted.

\section{Chromogenic in situ hybridization}

The CISH was performed using the ZytoDot ${ }^{\circledR} 2 \mathrm{C} \mathrm{SPEC}$ HER2/CEN 17 Probe and ZytoDot CISH Implementation Kit protocol (ZytoVision ${ }^{\circledR}$; ZytoDot ${ }^{\circledR}$ are trademarks of ZytoVision $\mathrm{GmbH})$. Briefly, the sections of the sequential TMA sections were dewaxed in $\left(70^{\circ} \mathrm{C}\right.$ for 10 minutes $)$ and rehydrated in xylene and ethanol. After deparaffinization, the slides were incubated in Heat Pretreatment Solution EDTA at a temperature $\geq 98^{\circ} \mathrm{C}$ for 15 minutes, followed by transferring slides immediately to $\mathrm{dH} 2 \mathrm{O}$ at the room temperature $\left(15-30^{\circ} \mathrm{C}\right)$. After washing in $\mathrm{dH} 2 \mathrm{O}$ (three times for 2 minutes), enzyme digestion was carried out by applying $100 \mathrm{ml}$ of digestion enzyme (pepsin solution with $0.05 \%$ sodium azide and detergent) to the slides for 5 minutes at the RT and humidity slide box. The slides were then washed with $\mathrm{dH} 2 \mathrm{O}$ and dehydrated with graded ethanols. Then, $15 \mu$ l of the ready-to-use HER 2 probe (Reagent C) was applied to the center of the $22 \times 22 \mathrm{~mm}$ slides, which were covered with coverslips and sealed with rubber cement. The sections were denatured on a thermal plate at $95^{\circ} \mathrm{C}\left( \pm 1^{\circ} \mathrm{C}\right)$ for 5 minutes and hybridization was followed by overnight incubation $(10-18 \mathrm{hrs})$ at $37^{\circ} \mathrm{C}$ $\left( \pm 1^{\circ} \mathrm{C}\right)$ in a humidity chamber.

After hybridization, the slides were washed with 0.5 SSC (standard saline citrate; $2-3 \mathrm{~min}$ at $70^{\circ} \mathrm{C}$ ), followed by three washes in $\mathrm{dH} 2 \mathrm{O}$ (at RT). The probes were detected by sequential incubations with CAS-BlockTM (10 min at $\mathrm{RT}$ ), mouse anti-digoxigenin (ready to use, $30 \mathrm{~min}$ at RT), goat anti-mouse HRP polymer conjugate (30 $\mathrm{min}$ at RT and humidity chamber) and diaminobenzidine chromogen (30 min at RT and humidity chamber). The tissue sections 
were lightly counterstained with hematoxylin, dehydrated in graded EtOH and immersed in xylene. A positive and a negative control sample (tumors with and without HER-2 amplification in CISH were included in every hybridization batch.

\section{Assessment of IHC and CISH results}

The samples were analyzed using the standard criteria for HER2 positivity. The IHC stain was scored by the standardized gastric cancer criteria (HercepTest ${ }^{\mathrm{TM}}$ ). For IHC, a negative score was considered when no membrane staining was observed, or when membrane staining was observed in less than $10 \%$ of the tumor cells $(0)$, or when faint or barely perceptible membranous reactivity was seen in $10 \%$ or more of the tumor cells while cells were reactive only in part of their membrane $(1+)$. Score $2+$ (equivocal) was given when weak to moderate complete, basolateral or lateral membranous reactivity was noted in $10 \%$ or more of the tumor cells, and score $3+$ (positive) was given when strong complete, basolateral or lateral membranous reactivity was noted in $10 \%$ or more of the tumor cells (Ruschoff et al., 2012; Madjd et al., 2014). For CISH assessments, the total numbers of HER 2 and CEP17 signals were counted in at least 20 tumor cell nuclei in 2 different areas a HER2 ratio of less than 2 was defined as negative for HER2 amplification and ratio of equal or more than 2 was defined as positive on average per cell (Ruschoff et al., 2012).

\section{Statistical analysis}

Statistical analyses were performed using the SPSS statistical software package version 20 (SPSS, Chicago, IL, USA). Pearson's $\chi^{2}$ and Pearson's R test were used to analyze the significance of correlation between the expression of HER2 and clinicopathological parameters. A p-value of $\leq 0.05$ was considered significant.

\section{Evaluation of immunostaining}

The immunostained tissue arrays were evaluated using a semi-quantitative scoring system (by NR) after a series was observed on a multi-headed microscope by two other observers (MM and NR) in a coded manner without any previous knowledge of the clinical or pathological parameters of the patients. The ambiguous cases were re-analyzed by two observers and consensus was reached with the second pathologist.

\section{Results}

Study population and demographic features of the patients

All 101 gastric tumor samples which were included in the present study were assessed by the IHC and CISH techniques. The clinical parameters are summarized in. The mean age of all patients in the present study was $60.13 \pm 11$ years (ranged between 32 and 82 years), whereas the majority $(53.5 \%)$ of the patients were between 61-82 years of age. There were 71 men $(70.5 \%)$ and 30 women $(29.5 \%)$. Of 101 samples, 62 cases $(61.40 \%)$ were intestinal subtype (according to Lauren`s classification), $30(30.70 \%)$ were diffuse subtype and 8 cases $(7.90 \%)$ were mixed carcinoma. The pathology grades of the patients were available and classified as follows: 56 (55.5\%) samples showed poorly differentiated, 25 (25.5\%) showed well-differentiated and 20 cases $(19 \%)$ had moderately differentiated histopathology. Of this series, 86 cases $(85.5 \%)$ were located in the stomach while 15 cases $(14.5 \%)$ were located in the gastroesophageal junction. Nodal involvement was present in 68 cases $(67.3 \%)$. Also, in terms of stage, 7 cases $(6.9 \%)$ were stage IA, 11 cases $(10.9 \%)$ stage IB, 29 cases $(28.7 \%)$ stage II, 31 cases (30.7\%) stage IIIA and 23 cases $(22.8 \%)$ were stage IIIB. The average size of the tumor was $5.35 \mathrm{~cm}$ ranging from 0.5 to $12 \mathrm{~cm} .41$ cases $(40.6 \%)$ were $5>\mathrm{cm}$ in the greatest diameter and 60 cases $(59.4 \%)$ were equal or more than 5 $\mathrm{cm}$ in the greatest diameter.

Tissue arrays consisting of 101 gastric, as well as gastroesophageal junction carcinomas were screened and evaluated for IHC staining of HER-2/neu. Out of 101gastric carcinomas, 13 cases (12.9\%) were HER-2/ neu positive in IHC staining (3+); 15 cases $(14.9 \%)$ had IHC score of $2+$, which should be further evaluated by chromogenic in situ hybridization (CISH) methods; 20 cases $(19.8 \%)$ had IHC score of $1+$ and 53 cases $(52.5 \%)$ had score of 0 for HER-2/neu staining. The latter 2 groups were considered negative.

\section{Evaluation of HER2 IHC scoring parameters for gastric cancer}

The analysis of tissue microarray-based immunohistochemistry staining demonstrated that the expression of HER2 was mainly localized in the cell membrane area of the tumors. The scoring method, based on the intensity of the staining, indicated that of 101 gastric tumors, $73(72.3 \%)$ cases showed negative $(0,+1)$ staining, $15(14.9 \%)$ showed equivocal $(+2)$ staining, and positive (+3) staining was detected in $13(12.8 \%)$ cases. Pearson Chi-Square test was used to compare the HER2 expression between all the gastric tissues in terms of intensity. A significant direct correlation was detected between the expression of HER2 in terms of the intensity of staining with the pathological grade, tumor type, tumor size and nodal involvement $(\mathrm{p}=0.015, \mathrm{P}=0.041, \mathrm{p}=0.038$ and $\mathrm{P}$ $=0.071$, respectively).

\section{Clinicopathological associations}

The analysis of tissue microarray-based immunohistochemistry staining demonstrated that HER-2/neu amplification had a significant correlation with the pathological grade $(\mathrm{P}$-value $=0.015)$, tumor type $(\mathrm{P}$-value $=0.041)$ and tumor size $(\mathrm{p}=0.038)$. In addition, HER-2/neu amplification had a trend association with lymph node involvement $(\mathrm{P}$-value $=0.071)$. It indicated that the overexpression of HER2 was significantly higher in poorly differentiated grade tumors, moderate in moderately differentiated grade samples and weaker in well differentiated gastric tissues. Also, this correlation was stronger in patients with the intestinal type of gastric adenocarcinoma $(\mathrm{p}=0.041)$, which was present in $76.9 \%$ of the intestinal type gastric adenocarcinomas. However, only 2 of the diffuse $(15.3 \%)$ and one of the mixed/ anaplastic carcinomas $(7.8 \%)$ showed gene amplification $(\mathrm{p}=0.041)$. The tumor size of the gastric adenocarcinomas 


\section{Nasser Rakhshani et al}

was compared with the HER2 expression using Pearson Chi-Square test (p-value $=0.038)$. The tumor size was categorized as diameter of $5>$ and $5 \leq \mathrm{cm}$, constituting $41(40.6 \%)$ and $60(58.4 \%)$, respectively. Our statistical analysis revealed that HER-2/neu overexpression was significantly higher in the larger tumors than the smaller ones $(\mathrm{p}>0.038)$. Positive nodal involvement tumors had rather association $68(67.5 \%)$ with HER-2/neu amplification than negative lymph node involvement tumors.

No correlation was found between the HER-2/neu overexpression and age, gender, tumor stage and tumor location.

\section{Assessment of CISH in gastric tumors}

Immunohistochemical testing is the primary method of choice to determine HER2 status in gastric cancer. CISH is restricted to all cases that have HER2 expression (IHC positive). CISH was performed according to the manufacturers' recommendations with ratios equal or above 2.0 being considered amplified (Positive). In particular, from 101 gastric samples, 17 (16.8\%) cases were amplified and the rest $(82.2 \%)$ were not amplified. Our statistical analysis demonstrated that the clinical features of the patients significantly correlated with tumor size and tumor type ( $\mathrm{p}=0.018$ and 0.058 , respectively) (Table1). Comparing the HER2 amplification with tumor type, according to $\mathrm{CISH}$ results, revealed that from 17 amplified samples $14(82.3 \%)$ were from intestine cases, $2(11.7 \%)$ from mixed carcinomas and only one sample from diffused type. Tumor size was the other feature which had a significant direct correlation $(\mathrm{p}=0.018)$ with HER2 amplification. Of $17 \mathrm{CISH}$ positive cases, 13 (76.4\%) had more than $5 \mathrm{~cm}$ diameter, and this indicates that the amplification of HER2 was higher in the large tumors (Table 1).

Table 1. Tumour Sample Characteristics and Association of HER2 Overexpression(CISH) with Clinicopathological Features in Gastric Samples (Pearson $\chi^{2}$ )

\begin{tabular}{|c|c|c|c|c|}
\hline \multicolumn{2}{|c|}{ Histopathological parameter } & \multicolumn{2}{|c|}{ CISH(N) } & \multirow[t]{2}{*}{$\mathrm{p}$ value } \\
\hline & & $2 \geq(\mathrm{Neg})$ & $2<(\operatorname{Pos})$ & \\
\hline \multirow[t]{2}{*}{ Age } & $\leq 60$ & 45 & 10 & 0.434 \\
\hline & $\geq 60$ & 39 & 7 & \\
\hline \multirow[t]{2}{*}{ Gender } & Male & 60 & 11 & 0.399 \\
\hline & Female & 24 & 6 & \\
\hline \multirow[t]{2}{*}{ Location } & Gej & 13 & 2 & 0.509 \\
\hline & Stomatch & 71 & 15 & \\
\hline \multirow[t]{3}{*}{ Grade } & Moderate & 15 & 5 & 0.411 \\
\hline & Poor & 49 & 7 & \\
\hline & Well & 20 & 5 & \\
\hline \multirow[t]{5}{*}{ Stage } & IA & 6 & 2 & 0.862 \\
\hline & IB & 9 & 1 & \\
\hline & II & 23 & 6 & \\
\hline & IIIA & 27 & 5 & \\
\hline & IIIB & 19 & 3 & \\
\hline \multirow[t]{3}{*}{ Tumor type } & Intestinal & 49 & 14 & 0.058 \\
\hline & Diffuse & 29 & 1 & \\
\hline & Mixed & 6 & 2 & \\
\hline \multirow[t]{2}{*}{ Tumor size } & $>5$ & 37 & 4 & 0.018 \\
\hline & $\leq 5$ & 47 & 13 & \\
\hline \multirow[t]{2}{*}{ Nodal involvement } & Positive & 57 & 11 & 0.516 \\
\hline & Negative & 27 & 6 & \\
\hline
\end{tabular}

\section{Concordance between IHC and CISH}

IHC analysis strictly following the modified gastric cancer testing protocol (Hofmann et al., 2008) showed overexpression of HER2 (IHC $3+)$ in $12.8 \%(n=13)$ of the gastric cancer samples. Within the IHC 3+ group, $76.9 \%$ of the tumors were intestinal-type adenocarcinomas, $15.3 \%$ diffuse-type adenocarcinomas and $7.8 \%$ were mixed adenocarcinomas tissues. Among the IHC 3+ group, $46.2 \%$ of the samples were poorly differentiated, $30.7 \%$ moderately differentiated and $23.1 \%$ were well differentiated cases. Concordance between IHC and CISH was $76.9 \%$, with all 13 IHC $3+$ cases also being CISH positive. HER-2 CISH analysis using the ZytoDot CISH Implementation Kit protocol resulted in a HER-2 positivity rate of $16.8 \%(\mathrm{n}=17)$. Thus, $7 \mathrm{CISH}+$ samples were determined to be negative by IHC (IHC 0 , IHC 1+) or equivocal (IHC 2+), using the gastric cancer scoring system

\section{Discussion}

HER-2/neu, as a prognostic marker and a targeted therapy for cancers of the gastrointestinal tract, has been of interest to many researchers recently (Tanner et al., 2005). Some studies have reported high overexpression of HER2/ neu protein in the tumors of the upper gastrointestinal tract (Koeppen et al., 2001; Ross and McKenna, 2001). The evaluation of a large panel of solid human tumors for the overexpression of HER2 with a single immunohistochemical staining assay was the primary goal of this study. Interestingly, the incidence of HER2 overexpression in gastric adenocarcinoma in our study is identical to that reported in a very large previous study (Ooi et al., 1998; Koeppen et al., 2001).

Excellent correlation has been reported between the data obtained from TMA and the conventional blocks in a variety of tumor types. Also, it has been found that sampling with optimal cores was sufficient to accurately detect the clinicopathological correlations (Hofmann et al., 2008; Ruschoff et al., 2010).

In our study, HER-2/neu overexpression was evaluated by immunohistochemistry staining in a microarray of 101 cases of gastric and gastroesophageal junction carcinomas. The observed prevalence of HER-2/neu overexpression $(12.8 \%)$ in our study $(3+$ in $0-3+$ scoring system) was slightly lower than the range found in most of the other published studies, which is mostly between 15 to $25 \%$ (Yano et al., 2004). However, a range of 9 to $38 \%$ positivity for HER-2/neu in the gastrointestinal carcinomas has been reported in different studies (Ishida et al., 1994; Tokunaga et al., 1995).

The incidence of HER2 overexpression defined as a HER2 score of greater than 2 remains controversial in at least some of these categories. It should be pointed out that our study was based only on immunohistochemistry staining, and we considered only $3+$ staining as HER-2/ neu overexpression. The $2+$ results were considered as equivocal which should further be evaluated by CISH method, which in turn can increase the prevalence of HER-2/neu positivity.

This study also showed a significant correlation 
between pathological grade, tumor type and tumor size with HER-2/neu protein overexpression ( $\mathrm{p}>0.015$, $\mathrm{p}>0.041$ and $\mathrm{p}>0.038$, respectively). In addition, a trend correlation was observed between nodal involvement and HER2/neu overexpression ( $\mathrm{p}>0.071)$.

Our analysis demonstrated a statistically significant correlation between the expressions of HER2/neu with the pathological grade of the gastric tumors ( $>0.015)$. This study had $46.2 \%$ poorly differentiated cancers, $30.7 \%$ moderately differentiated cancers and $23.1 \%$ well differentiated cancers. This association was somewhat stronger in patients with the intestinal type of gastric adenocarcinoma ( $p>0.001)$. In 2004, Tanner and his colleagues found a positive relationship between the pathological grade and HER2/neu overexpression (Tanner et al., 2005).

In terms of tumor size and expression of HER $2 /$ neu, around $60 \%$ of the larger tumors were HER-2/neu positive ( $>0.038$ ). This finding was not consistent with some studies (Chen et al., 2006), while some other researchers did not find any relationship between tumor size and HER-2/neu overexpression (Ghaderi et al., 2002; Chen et al., 2006).

The majority of the published studies found HER-2/ neu overexpression to be significantly associated with the intestinal subtype of gastric cancer, according to Lauren`s classification (Gravalos and Jimeno, 2008). In our study, the prevalence rate of HER-2/neu positivity in intestinal subtype was higher than the diffuse and mixed subtypes (76.9\% vs. $15.3 \%$ and $7.8 \%$, respectively); and there was a significant direct correlation $(\mathrm{p}>0.041)$.

In 2006, Chen et al. found that HER-2/neu expression was correlated with the lymph node involvement $(\mathrm{P}<0.05)$. Our results have indicated a direct trend correlation between lymph node involvement and HER2/neu overexpression ( $p>0.071)$ (Chen et al., 2006).

In this study, HER-2/neu protein overexpression was not associated with the mean age of the patients at the time of the diagnosis, pathologic stage and tumor location.

Many factors may account for the variable rates of HER2/neu immune reactivity as well as the conflicting reports suggesting that they are associated with adverse clinical outcomes in some studies but not others. These factors include small study sample sizes, tissue fixation, processing, choice of primary antibodies, detection systems, methods of antigen retrieval and subjective scoring system.

In our project, the detection of HER 2 positivity in gastric cancer also showed a relatively high level of concordance $(76.9 \%)$ between the two tests of validity using IHC and CISH. In a large group study in 2010, Okines and his colleagues (Okines and Cunningham, 2010) reported 22\% HER $2 /$ neu overexpression in 3807 patients. In another recently published study using the same assays in gastric cancer specimens, concordance between the results was $86.9 \%$ (Yano et al., 2006). It indicated that our result (the level of concordance) are consistent with which reported by Yano et al (Yano et al., 2006). In addition, among gastric cancers, the level of expression of HER2/neu according to CISH test was significantly positively correlated with tumor size and tumor type ( $p>0.018$ and 0.058 , respectively),implying that HER2/neu overexpression was higher in intestine and large tumors. However, in most related studies no sufficient information was provided about the correlation of expression of HER2/neu with CISH and clinical features. In conclusion, this study showed HER-2/neu overexpression in $13 \%$ of gastric and gastroesophageal junction carcinomas, most of which occurred at tumors $\geq 5 \mathrm{~cm}$ in the greatest diameter, poorly differentiated grade tumors, intestinal type and positive lymph node involvement tumors. Nevertheless, no significant association was found between HER2/neu overexpression and other clinic-pathological criteria (age, sex, tumor location and stage). Because this was a retrospective study, we could not elaborate about HER-2/neu overexpression and tumor prognosis.

A limitation in our study was that the 2 groups (HER-2/ neu positive vs. HER-2/neu negative tumors) were very different in number ( 13 cases of HER-2/neu positive vs. 88 cases of HER-2/neu negative). This difference in number can potentially have a negative impact on the analysis of data (although the review of the literature showed that the method of other published studies were the same as this study). In order to avoid such a potential bias in the statistical analysis, an equal number of HER-2/neu positive and HER-2/neu negative gastric cancers should be compared in future studies.

\section{References}

Allgayer H, Babic R, Gruetzner KU,et al (2000). c-erbB-2 is of independent prognostic relevance in gastric cancer and is associated with the expression of tumor-associated protease systems. J Clin Oncol, 18, 2201-2209.

Chen B, Luo RC, Cui F, Qian XY (2006). Association of HER-2/ neu expression with prognosis of gastric cancer. Nan Fang Yi Ke Da Xиe Xue Bao, 26, 344-7.

Choi JS, Kim MA, Lee HE, Lee HS, Kim WH (2009). Mucinous gastric carcinomas. Cancer, 115, 3581-90.

Eltze E, Wulfing C,Von Struensee D, et al (2005). Cox-2 and Her $2 /$ neu co-expression in invasive bladder cancer. Int $J$ Oncol, 26, 1525-31.

Ghaderi A, Vasei M, Maleck-Hosseini SA, et al (2002). The expression of c-erbB-1 and c-erbB 2 in Iranian patients with gastric carcinoma. Pathol Oncol Res, 8, 252-6.

Gheytanchi E, Mehrazma M, Madjd Z (2014). Expression of Ki-67, p53 and VEGF in pediatric neuroblastoma. Asian Pac J Cancer Prev, 15, 3065.

Gravalos C, Jimeno A (2008). HER2 in gastric cancer: a new prognostic factor and a novel therapeutic target. Ann Oncol, 19, 1523-9.

Hanna WM, wok KK (2006). Chromogenic in-situ hybridization: a viable alternative to fluorescence in-situ hybridization in the HER2 testing algorithm. Mod pathol, 19, 481-7.

Hetzel DJ, Wilson TO, Keeney GL, et al (1992). HER-2/neu expression: a major prognostic factor in endometrial cancer. Gynecol Oncol, 47, 179-185.

Hirashima N, Takahashi W, Yoshii S, Yamane T, Ooi A (2001). Protein overexpression and gene amplification of c-erb B-2 in pulmonary carcinomas: a comparative immunohistochemical and fluorescence in situ hybridization study. Mod Pathol, 14, 556-62.

Hofmann M, Stoss O, Shi D, et al (2008). Assessment of a HER2 scoring system for gastric cancer: results from a validation 
study. Histopathology, 52, 797-805.

Ishida T, Tsujisaki M, Hanzawa Y, et al (1994). Significance of erb B-2 gene product as a target molecule for cancer therapy. Scandinavian J Immunol, 39, 459-466.

Keymoosi H, Gheytanchi E, Asgari M, Shariftabrizi A, Madjd Z (2013). ALDH1 in combination with CD44 as putative cancer stem cell markers are correlated with poor prognosis in urothelial carcinoma of the urinary bladder. Asian Pac J Cancer Prev, 15, 2013-20.

Kiessling R, Wei WZ, Herrmann F, et al (2002). Cellular immunity to the Her-2/neu protooncogene. Adv Cancer Res, 85, 101-44.

Koeppen HKW, Wright BD, Burt AD, et al (2001). Overexpression of HER2/neu in solid tumours: an immunohistochemical survey. Histopathology, 38, 96-104.

Madjd Z, Ramezani B, Molanae S, Asadi-Lari M (2012). High expression of stem cell marker ALDH1 is associated with reduced BRCA1 in invasive breast carcinomas. Asian Pac J Cancer Prev, 13, 2973-8.

Madjd Z, Akbari ME, Zarnani AH, et al (2014). Expression of EMSY, a novel BRCA2-link protein, is associated with lymph node metastasis and increased tumor size in breast carcinomas. Asian Pac J Cancer Prev, 15, 1783-9.

Mehrazma M, Madjd Z, Kalantari E, et al (2013). Expression of stem cell markers, CD133 and CD44, in pediatric solid tumors: a study using tissue microarray. Fetal Pediatr Pathol, 32, 192-204.

Okines AFC, Cunningham D (2010). Trastuzumab in gastric cancer. Eur J Cancer, 46, 1949-1959.

Ooi A, Kobayashi M, Mai M, Nakanishi I (1998). Amplification of c-erbB-2 in gastric cancer: detection in formalinfixed, paraffin-embedded tissue by fluorescence in situ hybridization. Lab invest, 78, 345-51.

Reichelt U, Duesedau P, Tsourlakis MCh, et al (2007). Frequent homogeneous HER-2 amplification in primary and metastatic adenocarcinoma of the esophagus. Mod Pathol, 20, 120-9.

Ross JS, McKenna BJ (2001). The HER-2/neu oncogene in tumors of the gastrointestinal tract. Cancer Investigation, 19, 554-68.

Ruschoff J, Dietel M, Baretton G, et al (2010). HER2 diagnostics in gastric cancer-guideline validation and development of standardized immunohistochemical testing. Virchows Archiv, 457, 299-307.

Ruschoff J, Hanna W, Bilous M, et al (2012). HER2 testing in gastric cancer: a practical approach. Mod Pathol, 25, 637-650.

Schuell B, Gruenberger T, Scheithauer W, Zielinski Ch, Wrba F (2006). HER 2/neu protein expression in colorectal cancer. BMC Cancer, $\mathbf{6}, 123$.

Shinohara H, Morita S, Kawai M, et al (2002). Expression of HER2 in human gastric cancer cells directly correlates with antitumor activity of a recombinant disulfide-stabilized antiHER2 immunotoxin. J Surg Res, 102, 169-177.

Slamon DJ, Leyland-Jones B, Shak S, et al (2001). Use of chemotherapy plus a monoclonal antibody against HER2 for metastatic breast cancer that overexpresses HER2. $N$ Engl J Med, 344, 783-92.

Smith I, Procter M, Gelber RD, et al (2007). 2-year follow-up of trastuzumab after adjuvant chemotherapy in HER2-positive breast cancer: a randomised controlled trial. The Lancet, 369, 29-36.

Takehana T, Kunitomo K, Kono K, et al (2002). Status of c-erbB-2 in gastric adenocarcinoma: a comparative study of immunohistochemistry, fluorescence in situ hybridization and enzyme-linked immuno-sorbent assay. Int J Cancer, 98, 833-7.

Tanner M, Hollmen M, Junttila TT, et al (2005). Amplification of
HER-2 in gastric carcinoma: association with Topoisomerase II $\alpha$ gene amplification, intestinal type, poor prognosis and sensitivity to trastuzumab. Ann Oncol, 16, 273-278.

Tapia C, Glatz K, Novotny H, et al (2007). Close association between HER-2 amplification and overexpression in human tumors of non-breast origin. Mod Pathol, 20, 192-198.

Thompson SK, Sullivan TR, Davies R, Ruszkiewicz AR (2011). Her-2/neu gene amplification in esophageal adenocarcinoma and its influence on survival. Ann Surg Oncol, 18, 20102017.

Tokunaga A, Onda M, Okuda T, et al (1995). Clinical significance of epidermal growth factor (EGF), EGF receptor, and c-erbb-2 in human gastric cancer. Cancer, 75, 1418-25.

Yano T, Ochiai A, Doi T, et al (2004). Expression of HER2 in gastric cancer: comparison between protein expression and gene amplification using a new commercial kit. J Clin Oncol, 22, 4053.

Yano T, Ohtsu A, Boku N, et al (2006). Comparison of HER2 gene amplification assessed by fluorescence in situ hybridization and HER2 protein expression assessed by immunohistochemistry in gastric cancer. Oncology Reports, 15, 65-71. 